\title{
Modeling of Transient Flow Mixing of Streams Injected into a Mixing Chamber
}

\author{
Dmytro M. Voytovych",Charles L. Merkle ${ }^{\dagger}$, Robert P. Lucht ${ }^{\ddagger}$ \\ Purdue University, West Lafayette, IN, 47907 \\ James R. Hulka ${ }^{\S}$ \\ Jacobs Sverdrup, Huntsville AL \\ Gregg W. Jones** \\ NASA Marshall Space Flight Center, Huntsville, AL 47907
}

\section{Introduction}

Ignition is recognized as one the critical drivers in the reliability of multiple-start rocket engines. Residual combustion products from previous engine operation can condense on valves and related structures thereby creating difficulties for subsequent starting procedures. Alternative ignition methods that require fewer valves can mitigate the valve reliability problem, but require improved understanding of the spatial and temporal propellant distribution in the pre-ignition chamber. Current design tools based mainly on one-dimensional analysis and empirical models cannot predict local details of the injection and ignition processes. The goal of this work is to evaluate the capability of the modern computational fluid dynamics (CFD) tools in predicting the transient flow mixing in pre-ignition environment by comparing the results with the experimental data. This study is a part of a program to improve analytical methods and methodologies to analyze reliability and durability of combustion devices.

In the present paper we describe a series of detailed computational simulations of the unsteady mixing events as the cold propellants are first introduced into the chamber as a first step in providing this necessary environmental description. The present computational modeling represents a complement to parallel experimental simulations ${ }^{1}$ and includes comparisons with experimental results from that effort.

A large number of rocket engine ignition studies has been previously reported. Here we limit our discussion to the work discussed in Refs. 2, 3 and 4 which is both similar to and different from the present approach. The similarities arise from the fact that both efforts involve detailed experimental/computational simulations of the ignition problem. The differences arise from the underlying philosophy of the two endeavors. The approach in Refs. 2 to 4 is a classical ignition study in which the focus is on the response of a propellant mixture to an ignition source, with emphasis on the level of energy needed for ignition and the ensuing flame propagation issues. Our focus in the present paper is on identifying the unsteady mixing processes that provide the propellant mixture in which the ignition source is to be placed. In particular, we wish to characterize the spatial and temporal mixture distribution with a view toward identifying preferred spatial and temporal locations for the ignition source. As such, the present work is limited to cold flow (pre-ignition) conditions. Representative conditions and flow rates are discussed in Ref. 1.

\section{Problem Specification and Computational Model}

The present unsteady, transient mixing simulations represent the modeling counterpart for experiments described elsewhere. ${ }^{1}$ Additional information is available in [2-Lucht JANNAF paper]. The focus of the combined experimental/computational program is to understand the unsteady mixing processes that occur in a liquid propellant rocket engine prior to ignition, with an emphasis on characterizing appropriate time

\footnotetext{
* Post-Doctoral Research Associate, School of Aeronautics and Astronautics, Member AIAA.

${ }^{\dagger}$ Reilly Professor of Engineering, Member AIAA.

${ }^{\ddagger}$ Professor, School of Mechanical Engineering, Member AIAA.

${ }^{\S}$ Engineering Specialist, MSFC Group, M/S ER32, NASA MSFC, AL, 35812; Senior Member, AIAA.

${ }^{* *}$ Combustion Specialist, M/S ER32, NASA MSFC, AL, 35812.
} 
scales and pertinent locations at which an ignition source would be most effective. The specific objective is to understand the variation of local mixture fraction as a function of time. In both the experiments and the computations, helium is used as a simulant for hydrogen and nitrogen is used as a simulant for oxygen. For this initial examination of the problem, both the experiments and computations used gaseous propellants as the simulation for a more complex problem that includes two-phase flow of oxygen.

The geometry is given in Figs. 1 and 2. Fig. 1 shows a sketch of the experimental configuration to give an overview of the geometry of interest, while Fig. 2 gives pertinent dimensions in the cross-plane. The configuration is composed of an injector assembly through which the propellants are introduced into a windowed mixing chamber. The injector contains three inlet channels that feed nitrogen and helium into the chamber as shown on the left side of Fig. 2. Each of these channels was $101.6 \mathrm{~mm}$ (4") long to ensure fully developed turbulent flow into the chamber. The central channel carried nitrogen and was $3.2 \mathrm{~mm}\left(1 / 8^{\prime \prime}\right)$ high while the two outer channels

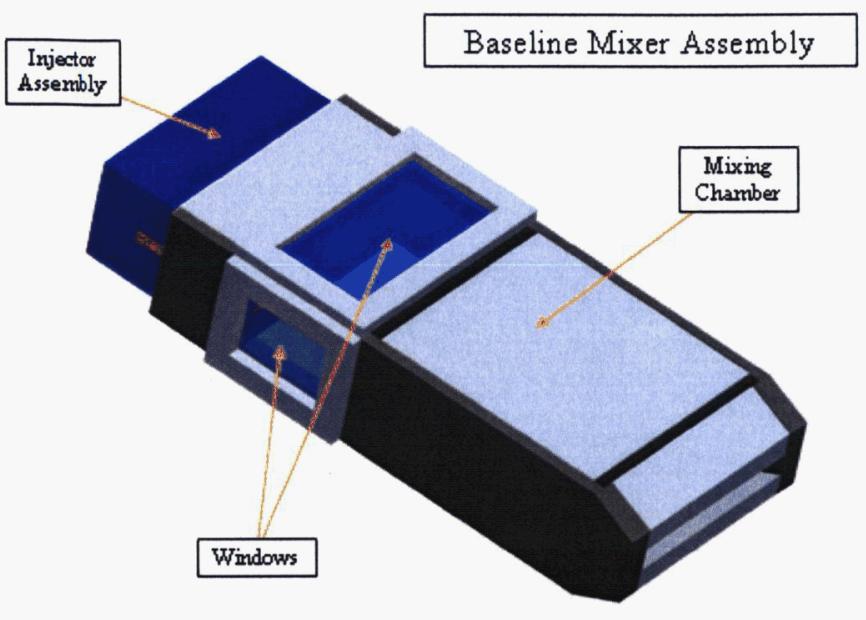

Figure 1. Injector-chamber configuration. Flow is from unner left to lower right. carried helium and were each $1.6 \mathrm{~mm}\left(1 / 16^{\prime \prime}\right)$ high. The three channels were separated by splitter plates of $1.6 \mathrm{~mm}(1 / 16 ")$ thickness. The overall dimensions of the chamber were $38.1 \mathrm{~mm}$ (1.5") high by $203.2 \mathrm{~mm}$ (8") long with a width (in the plane perpendicular to the sketch in Fig. 2) of $101.6 \mathrm{~mm}$ (4"). The three inlet slots were centered in the $38.1 \mathrm{~mm}(1.5$ ") upstream face with a back step between the helium channel and the outer chamber wall of $14.3 \mathrm{~mm}$ (9/16"). The three inlet slots extended the entire four inches across the chamber. The mixing chamber is terminated by a straight nozzle of length $25.4 \mathrm{~mm}$ (1") that converges to an exit height of $12.7 \mathrm{~mm}\left(0.5^{\prime \prime}\right)$ to provide a more positive exit condition for the subsonic outflow.

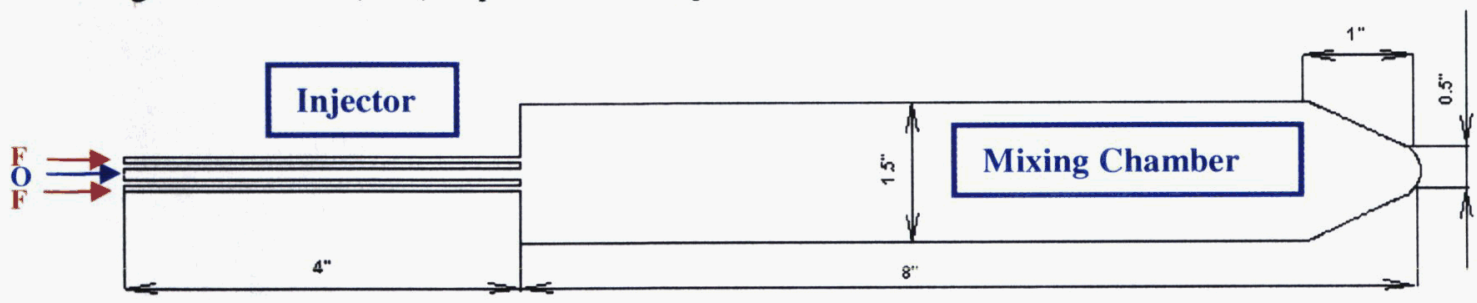

Figure 2: Dimensions of inlet channels and mixing chamber.

The experimental simulations started from a nitrogen (oxidizer simulant) lead into the chamber followed by helium (fuel simulant). Accordingly, the computations likewise started by a steady flow of nitrogen into which a transient flow of helium was introduced at the upstream end of the channels at time zero. Upon opening the helium valve, we follow the dynamics of the helium flow as it passes through the helium channel, emerges into the mixing chamber, transiently mixes with the nitrogen and finally produces a mixture of helium and nitrogen in the chamber. Of particular interest in the sequence is an understanding of the rate of change of the concentration of helium in the recirculating regions adjacent to the upstream face and outer walls. Both two-dimensional and three-dimensional computations have been conducted. The two-dimensional computations allow a careful study of grid sensitivity and an identification of fundamental physics, while the three-dimensional solutions address potential non-planar effects.

The nominal flow rates were $59.6 \mathrm{~g} / \mathrm{s}$ of N2 and $8.028 \mathrm{~g} / \mathrm{s}$ of He. The helium flow was split equally between the two fuel stimulant slots. The experiments were conducted with room temperature gasses with a chamber pressure of nominally one atmosphere. The converging section at the exit was unchoked. The mean velocities in the nitrogen and helium passages were $175.5 \mathrm{~m} / \mathrm{s}$ and $336.6 \mathrm{~m} / \mathrm{s}$, respectively, 
corresponding to a Mach number of 0.5 in the nitrogen and 0.35 in the helium. The Reynolds numbers were 38,600 in the nitrogen stream and 4650 in the helium stream. Steady state computations of the flow in the nitrogen passage on a grid of $200 \times 100$ verified that fully developed flow was reached, and that the pressure gradient agreed with classical turbulent channel flow computations. Unsteady helium computations were run to verify the accuracy of the helium flow transient and to check the times required for the helium to reach the chamber. The transient computations were initiated by 'breaking' a diaphragm near the upstream end of the helium channel that separated helium at the upstream plenum conditions from nitrogen at ambient conditions. The results indicate that the weak precursor shock from the diaphragm breakage reaches the chamber in $0.25 \mathrm{~ms}$, followed by the helium interface (which heralds the arrival of helium into the chamber) approximately $1 \mathrm{~ms}$ later. These times are short in comparison to the mixing times of interest. The nominal time to fill the helium plenum in the chamber at these flow rates was calculated to be $30 \mathrm{~ms}$. The following sections describe the steady nitrogen flow first and then discuss the mixing between the helium and the nitrogen.

The computations were based on time accurate solutions of the Navier-Stokes equations using a second-order finite-volume spatial discretization and three-point backward differencing for the temporal discretization. ${ }^{10}$ A dual-time method was used to circumvent approximate factorization errors. The code used for solving the equations has capabilities for an arbitrary unstructured grid. ${ }^{9}$ Most of the present solutions were based on quadrilateral (hexahedral) grids in the internal flow regions although triangular grids were used for portions of the external domains. The solutions were based upon a perfect gas equation of state and a k- $\omega$ turbulence model. ${ }^{8}$ The computational domain for all simulations was started at the upstream end of the injector and included the complete injector, the mixing chamber and an external domain outside the chamber which was used to enable improved downstream boundary conditions. The nominal grid sizes for the two-dimensional computations were 120,000 cells, while in the threedimensional simulations much coarser grid sizes of approximately 2.5 million cells were used.

\section{Steady Nitrogen Flowfield Characteristics}

The first step in simulating the transient mixing problem of interest is to establish the steady nitrogen flowfield into which the helium is injected. Although this steady flowfield appears to be quite routine, the computational solutions indicate three qualitatively different flow structures can exist. The simplest condition is a steady, symmetric jet emanating from the central channel with two recirculating flow regions adjacent to the face on either side of the jet. Solutions of this type were readily obtained by solving the symmetry problem in half the chamber or by simulating problems with smaller back-step regions and lower Reynolds numbers. However, when the complete chamber was computed at the conditions of interest, the jet invariably deviated to one side and attached stably to the wall where it remained until exiting the nozzle in an asymmetric manner. A third possibility was an unsteady solution in which the jet meandered through the chamber in a time-varying fashion, suggesting that no stable, steady solution existed for these conditions. Both the current experiments ${ }^{1}$ as well as observations reported in the literature ${ }^{11}$ suggested that all three of these conditions can be realized, depending on the parameters. In the present section we describe representative results from a large number of 'steady' flow nitrogen simulations.

\section{Symmetric Nitrogen Solutions}

To provide a global overview of the chamber flow patterns with only nitrogen flowing, the solution in the half chamber with a symmetry condition applied on the mid plane is shown first. The stagnation pressure upstream of the nitrogen channel was set to $1.2 \mathrm{~atm}$ for this case and the nozzle exit pressure to 0.5 atm. The reduced exit pressure resulted in a flow approximately twice the base rate and created a weak, supersonic shock-diamond pattern as the jet expanded into the chamber. Nevertheless, the computed solution provides a qualitative indication of the expected flow characteristics.

The streamlines superimposed on the Mach number contours in Fig. 3 show that the symmetric jet induced a long, narrow recirculation vortex. (For clarity this symmetric solution has been reflected about

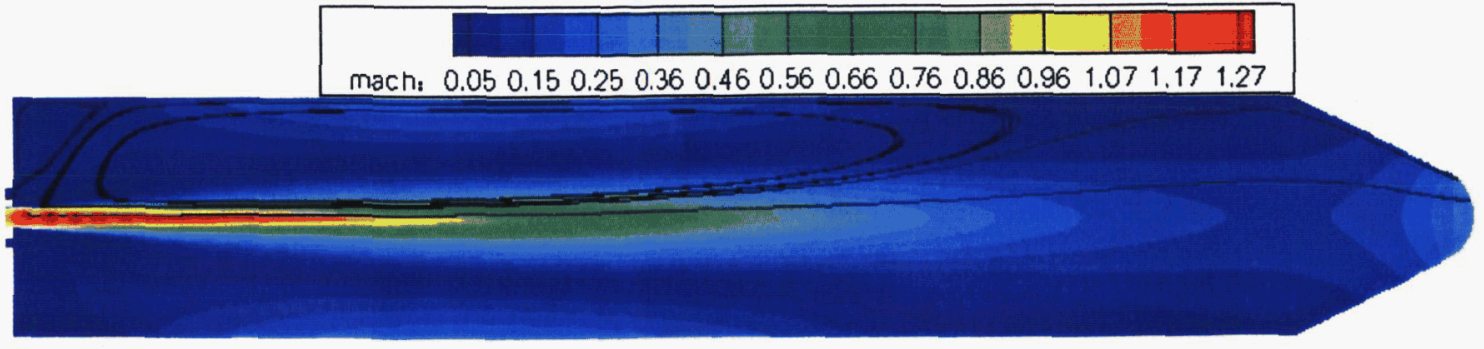

Figure 3. Mach number contours and streamlines for symmetric solution (reflected about the 
the symmetry plane to show the entire cross-section of the mixing chamber.) Later solutions without a symmetry condition indicated that these vortices destabilized the jet and tended to cause it to attach to one wall in Coanda-like fashion. In addition there was a small vortex pair in the upper and lower corners of the mixing chamber near the injector face. For this solution, the constant pressure downstream boundary condition was enforced on a curved arc shown at the nozzle exit in Fig. 3. The shape of the arc was taken from constant pressure contours in companion solutions that included an external domain and provides an approximate means for accounting for this effect.

\section{Full-Domain Nitrogen Solutions}

Full domain solutions in which both the upper and lower halves of the chamber were computed without invoking a symmetry condition resulted in a flow pattern much different than that shown in Fig. 3 . A representative solution for the full domain based on exactly the same conditions as those used for the symmetric solution in Fig. 3 (except that the full domain was computed) is given in Fig. 4 . Again, the upstream stagnation pressure was $1.2 \mathrm{~atm}$ and the exit pressure was $0.5 \mathrm{~atm}$. The result clearly shows that the flow is not symmetric but attaches to the upper wall shortly downstream of the injector face and remains attached to the wall until it exits the nozzle. This flow pattern resulted in one small recirculation zone on the upper side of the jet, and a large, weak recirculation zone that covered most of the volume of the mixing chamber on the other side of the jet. Additional smaller recirculation regions appeared in the corners.
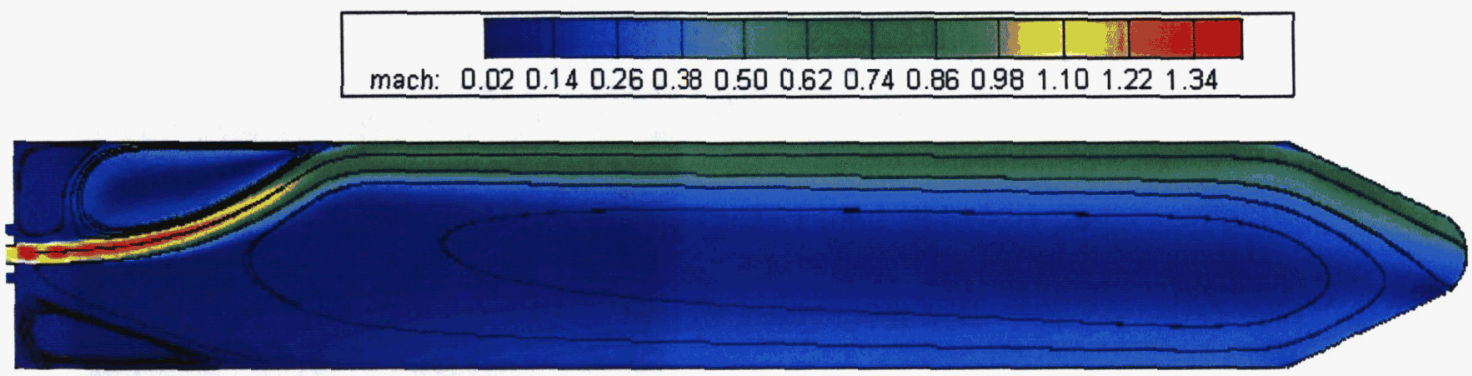

Figure 4. Mach number contours and streamlines for full domain solution. Exit pressure 0.5 atm. Inlet stapnation nressure 1.2 atm.

The geometry of the present mixing chamber is completely symmetric about the mid-plane, and there is no reason to expect that the flow should prefer to attach to the top wall as compared to the bottom wall. To demonstrate that the flow will attach to either wall, we show in Fig. 5 the results of two different solutions for the base geometry. In one of these solutions, the flow attached to the top wall as in Fig. 4, while in the other, it attached to the bottom wall. These computations are identical apart from a minor difference in the initial condition. The two solutions are also identical except for the direction of the asymmetry, and are both very stable to flow perturbations because the pressure in the small recirculation region is slightly lower than that on the opposite side of the jet, providing the force needed to keep the jet attached to the wall. The solution appears to choose to attach to one wall relatively early in the convergence process, and once it has attached, becomes very stable. Also note that the solutions in Fig. 5 are for an upstream stagnation pressure of $1.2 \mathrm{~atm}$ and an exit pressure of $1.0 \mathrm{~atm}$ corresponding to the base flow conditions given above. The fact that two different back pressures give similar results is a further demonstration of the tendency toward asymmetric solutions. The resulting exit Mach number of the nitrogen jet as it emerges from the injector passage is now about 0.5 , rather than the sonic condition observed in Fig. 4.

In addition to these solutions, a large number of computations for various flow rates and mixing chamber heights indicated that this asymmetric solution was far more common than the symmetric solution, and that, once it attached to the wall, it remained very stable. Symmetric solutions were obtained only for mixing chamber heights one-fourth the present one at Reynolds numbers approximately a factor of ten smaller. Finally, computations run with the commercial code, Fluent, gave similar asymmetric results as 
did solutions by West ${ }^{12}$ using a NASA in-house code. Flow visualization results from the companion experiments ${ }^{1}$ also suggest that off-axis flows are often encountered as later comparisons show.

Before leaving these nitrogen-only, steady flow solutions, we note that in all cases, the computational domain included the complete length of the inlet channels in addition to the mixing chamber. An external domain downstream of the chamber was also included in most cases to provide a more robust downstream boundary condition. The nitrogen flow rate was set by specifying the stagnation temperature $(300 \mathrm{~K}$ in all cases) and pressure (as specified above) at the upstream end of the center channel shown in Fig. 2. For the nitrogen-only calculations, the two outer helium channels were also filled with nitrogen and included in the computation, but their upstream ends were blocked so there was no net flow through them. The computational grid included approximately 120,000 cells with stretching near the channel and chamber walls to a value of $y^{+} \approx 1$ on all walls.
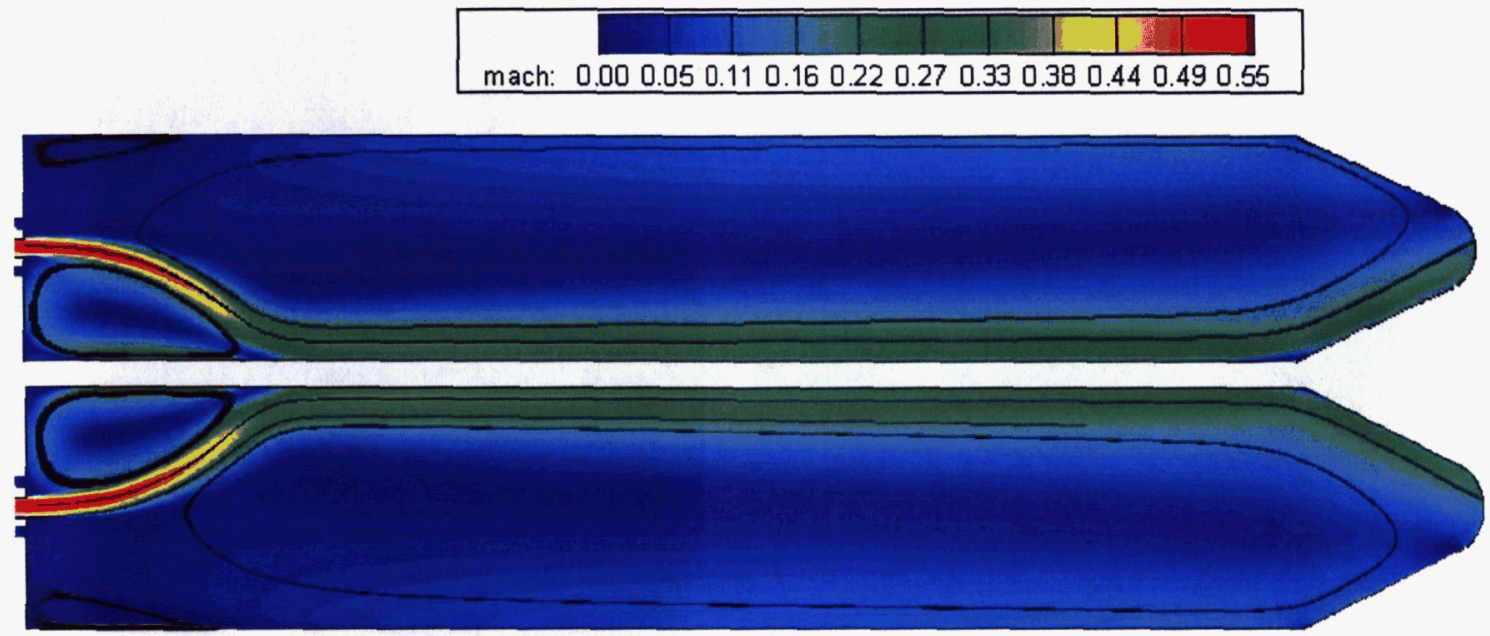

Figure 5. Mach number contours and streamlines for full domain solution for two initial conditions. Fixit nressure 1.0 atm. Inlet stagnation nressure $1.2 \mathrm{~atm}$.

\section{Two-Dimensional Transient Helium/Nitrogen Mixing Simulations}

The previous section indicates that the steady flow of nitrogen through the mixing chamber results in an asymmetric flowfield. In the present section we detail the characteristics of the transient mixing between a helium stream that is initiated at time zero and this (initially) steady nitrogen flow. The computations in this section are two-dimensional. Three-dimensional results are presented in the following section to assess effects of finite chamber aspect ratio on the predicted flowfields. We first present results showing the transient solution in the helium channels followed by results showing the propagation of helium through the mixing chamber. We conclude this section by making some comparisons between nitrogen mixing into nitrogen and nitrogen mixing into helium to demonstrate molecular weight effects. An external domain was used for all transient calculations.

\section{Transient Helium Flow in Channels}

The transient solutions start by breaking a diaphragm near the upstream end $(5 \mathrm{~mm})$ of the helium channel. The fluid on the upstream side of the diaphragm is quiescent helium at a pressure and temperature corresponding to the desired stagnation conditions for helium on the upstream end of the channel. The fluid on the downstream side of the diaphragm is nitrogen at conditions determined by the steady-flow nitrogen calculation. Calculations based upon the specified flow rate and the plenum volume in the experiment indicate a plenum fill time of approximately $30 \mathrm{~ms}$, a time that agrees well with experimental evidence of the time between the signal to the helium valve and the first appearance of helium in the 
chamber. Since the computation ignores the plenum fill time, the 'valve' opening time in the computations is arbitrarily set at $30 \mathrm{~ms}$ to enable comparison with experiment.

An $x-t$ diagram of the dynamics of the flow in the channel is given in Fig. 6. The plot on the left shows the $u, u+c$, and $u-c$ characteristics for the first $250 \mu \mathrm{s}$ along the entire length of the channel, while the plot on the right shows the corresponding pressure contours. As soon as the diaphragm is broken, a weak shock corresponding to the pressure difference between the quiescent nitrogen in the channel (nominally $1 \mathrm{~atm}$ ) and the helium at upstream stagnation conditions $(1.1 \mathrm{~atm})$ propagates through the channel and reaches the chamber just after the first $250 \mu$ s time-frame shown in the figure. This is clearly seen in both plots of Fig. 6. When this precursor shock reaches the chamber, the residual nitrogen that was trapped in the helium chamber will emerge first, followed by the helium at a later time. The helium-nitrogen interface (which moves at the particle speed instead of the acoustic speed) is also visible in the plot on the left and indicates that at $250 \mu \mathrm{s}$ the helium has moved less than one-fourth of the distance through the channel, so that the first helium should appear after approximately $1 \mathrm{~ms}$ following valve opening.

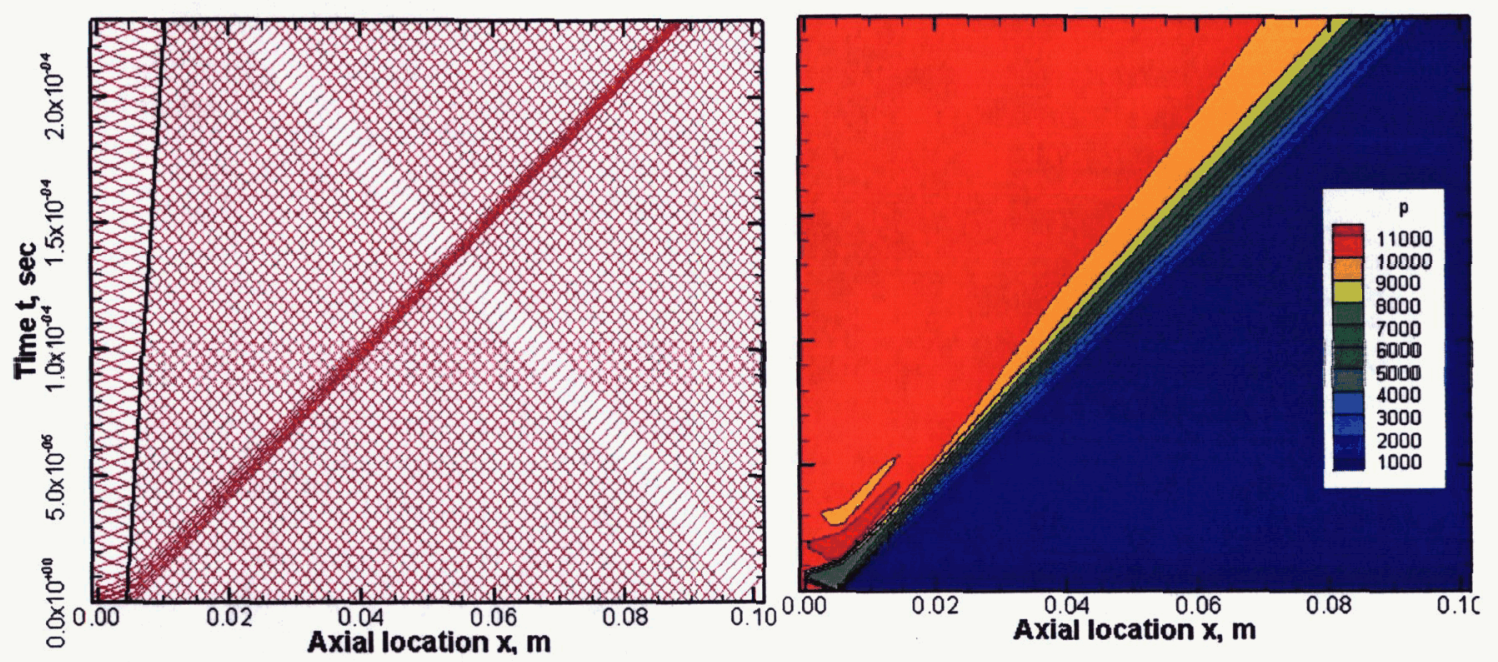

Figure 6. $x-t$ diagram for flow through helium channel. Left: $u, u+c$, and $u-c$ characteristics. Right Pressure contours. Time. $30<t<30.250 \mathrm{~ms}$.

\section{Transient Helium Flow in Chamber}

A series of snapshots of the transient solution showing the instantaneous contours of helium concentration in the chamber are shown in Fig. 7 for times $t=31.3,32.2,35,40,50,65$, and $80 \mathrm{~ms}$. Only the mixing chamber and part of the external domain are shown, the injector inlet channels are ommitted . The steady nitrogen flow into which these helium streams are injected corresponds to the one in the lower half of Fig. 5 where the flow is attached to the upper wall. This asymmetric nitrogen flow is mirrored clearly in the helium transient. The first frame at the top of Fig. 7 shows the helium enters through the upper chamber sooner than through the lower chamber because the pressure in the small recirculation region is slightly lower than that on the other side. The helium from the upper jet appears approximately 1 $m s$ earlier than that in the lower one. Similar differences in the time the helium emerges from the two channels are also observed in the experiment. In some experiments, this difference is approximately $1 \mathrm{~ms}$ while in others it is nearly $3 \mathrm{~ms}$. 


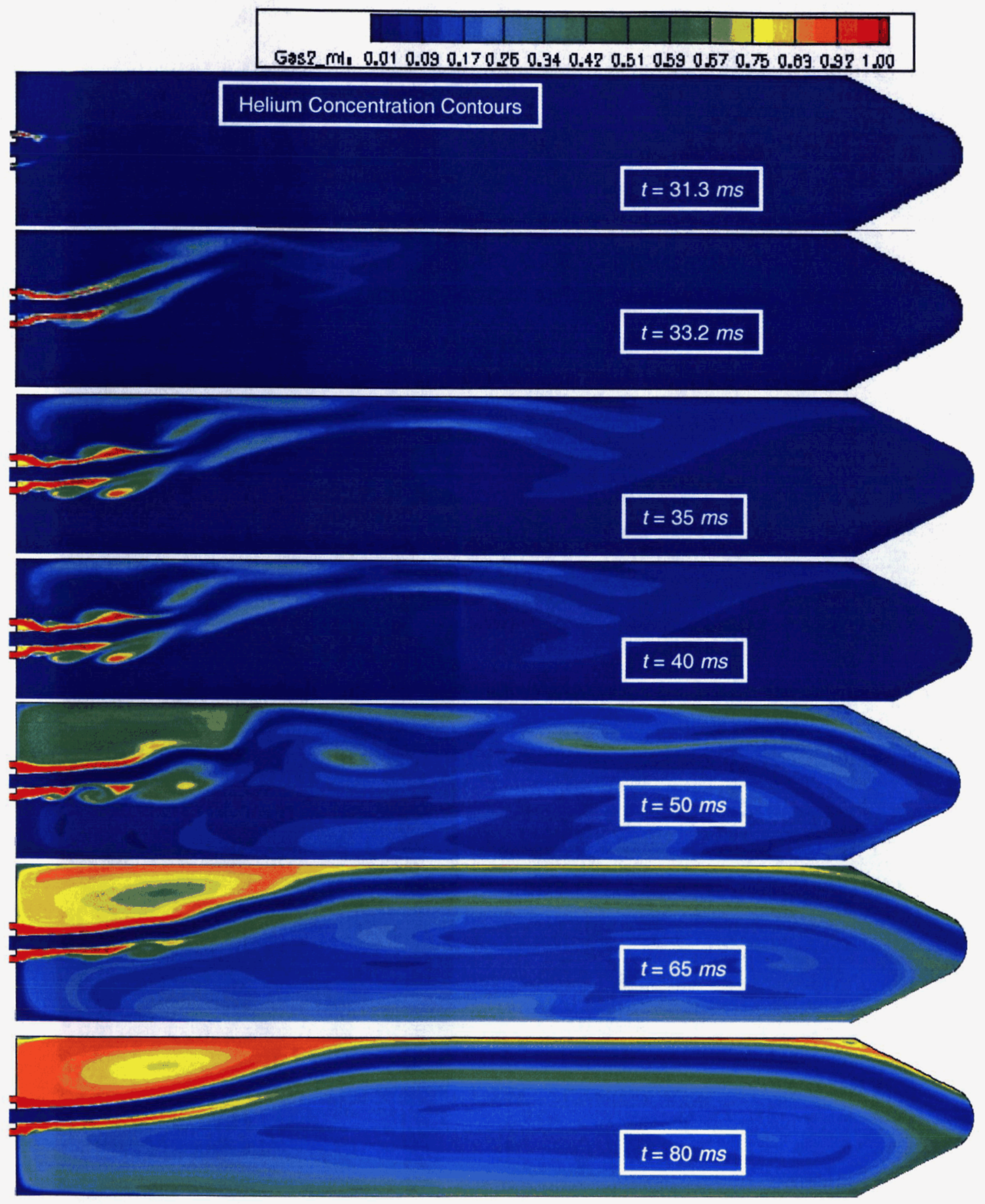

Figure 7. Unsteady helium concentration contours at different times.

As time progresses $(t=33.2$ and $35 \mathrm{~ms}$ ) the initial jets of helium are observed to outline the asymmetric nitrogen flow and to progress downstream through the chamber. At the $35 \mathrm{~ms}$ condition, the flow from the upper channel also begins to enter the upper recirculation region to start filling it and displacing the nitrogen initially there. Note that small vortices develop on the outer side of the helium stream by the shear between the lighter helium and the nitrogen in the surrounding mixing chamber at the earlier times (up to $50 \mathrm{~ms}$ ). These vortices generate packets of helium that propagate downstream along the main nitrogen jet. At later times as the upper recirculation region fills with helium, the rollup on this outer edge decreases dramatically. 
At the later times $(t=65$ and $80 \mathrm{~ms})$, the upper recirculation region is seen to be nearly completely filled with helium and the reattachment point of the nitrogen/helium jet moves from approximately $40 \mathrm{~mm}$ downstream of the injector face to $80 \mathrm{~mm}$. The path of the nitrogen jet is also clearly marked by the two adjoining helium streams in these last two plots.

Clearly, the time required to completely fill the large (lower) recirculation region with helium is considerably longer than the present computations, but the results give a good indication of the manner in which the initial part of the transient progresses. In particular the stoichiometry of the upper recirculation region has passed through 'flammability' limits, while that in the lower recirculation zone has yet to reach these levels. Extrapolating ahead, the results suggest that, eventually, the flow will reach steady state again with nitrogen flowing through a chamber filled with helium.

\section{Effects of Molecular Weight on Mixing}

In the later portion of the mixing process described above, streams of nitrogen and helium flow into a chamber whose background gas is helium, while earlier they flow into a chamber filled with nitrogen. The presence of a lighter background gas should diminish the tendency for the incoming fluid to attach to the wall. To assess the magnitude of this effect, we compare the results of two auxiliary transient simulations. In the first, a nitrogen jet is introduced into a chamber filled with quiescent helium, while in the second, a nitrogen jet is introduced into one filled with quiescent nitrogen. In both cases, the incoming nitrogen is introduced through the middle channel of the three-channel injector described above. A series of snapshots of the time history of these two calculations are presented in Figs. 8 and 9. Fig. 8 shows the results for nitrogen into helium while Fig. 9 shows nitrogen into nitrogen. In both figures the contours show the instantaneous concentration of the entering nitrogen. Instantaneous contours are shown at $32,34,36,38$, 40 and $50 \mathrm{~ms}$ for both calculations.

Comparison of the solutions at $t=32 \mathrm{~ms}$ indicates that the nitrogen moves through the lighter helium (Fig. 8) much more rapidly than through the heavier nitrogen (Fig. 9). This faster movement continues throughout the solution. At $t=34 \mathrm{~ms}$, for example, a substantial amount of nitrogen in the helium background gas has traversed the mixing chamber and exited through the nozzle, while the nitrogen into nitrogen does not reach the external domain until $t=38 \mathrm{~ms}$.

The dynamics of the jet streams in the two background media are considerably different, as can be seen by comparing the two figures. When the nitrogen flows into the heavier media, it produces a much stronger starting vortex that rolls up in front of the jet, whereas in the lighter media almost no starting vortex is observed and the jet proceeds along the centerline until it is nearly half way through the mixing chamber. After it reaches the mid-point of the chamber, the nitrogen-in-helium jet begins to deviate widely from the centerline as the solution becomes highly asymmetric. The initial point of attachment to the wall is much further downstream than that observed in the steady nitrogen solution, but at the later time the background helium is flushed out of the chamber and the solution appears to approach the steady nitrogen solutions shown earlier. It is important to note, however, that at later times, this nitrogen-into-helium solution should be expected to approach the steady nitrogen solution shown in Fig. 5. The nitrogen-intonitrogen solution begins to deviate from the centerline before it has traversed as large a fraction of the chamber than for the case where the nitrogen is flowing into helium, although the actual elapsed time is about the same. The initial point at which the nitrogen-nitrogen solution attaches to the wall is also much closer to the inlet face (and to the attachment point of the steady nitrogen solution in Fig. 5) than for the nitrogen-helium case. 

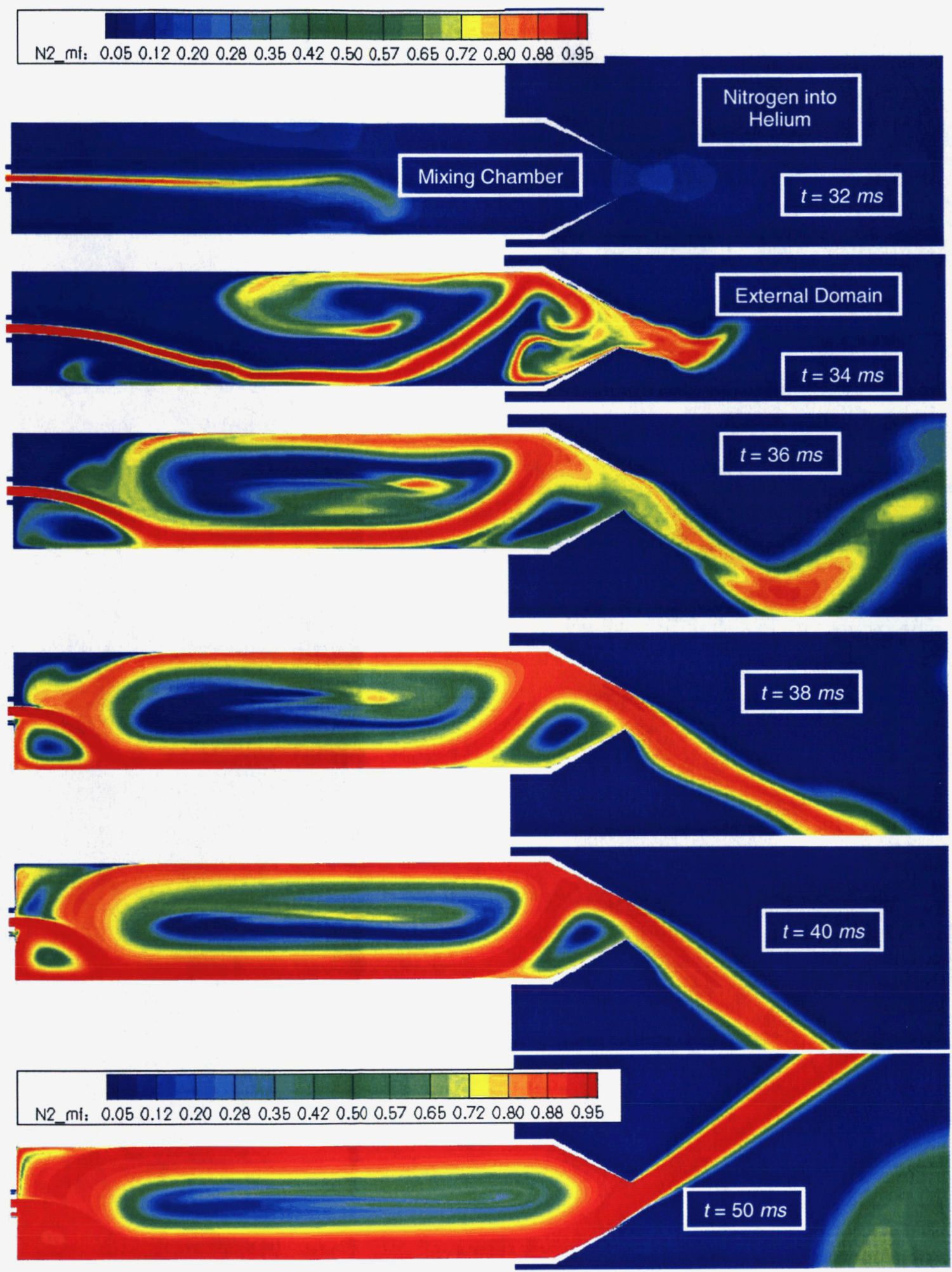

Figure 8. Various times of transient mixing of nitrogen injected into helium. 


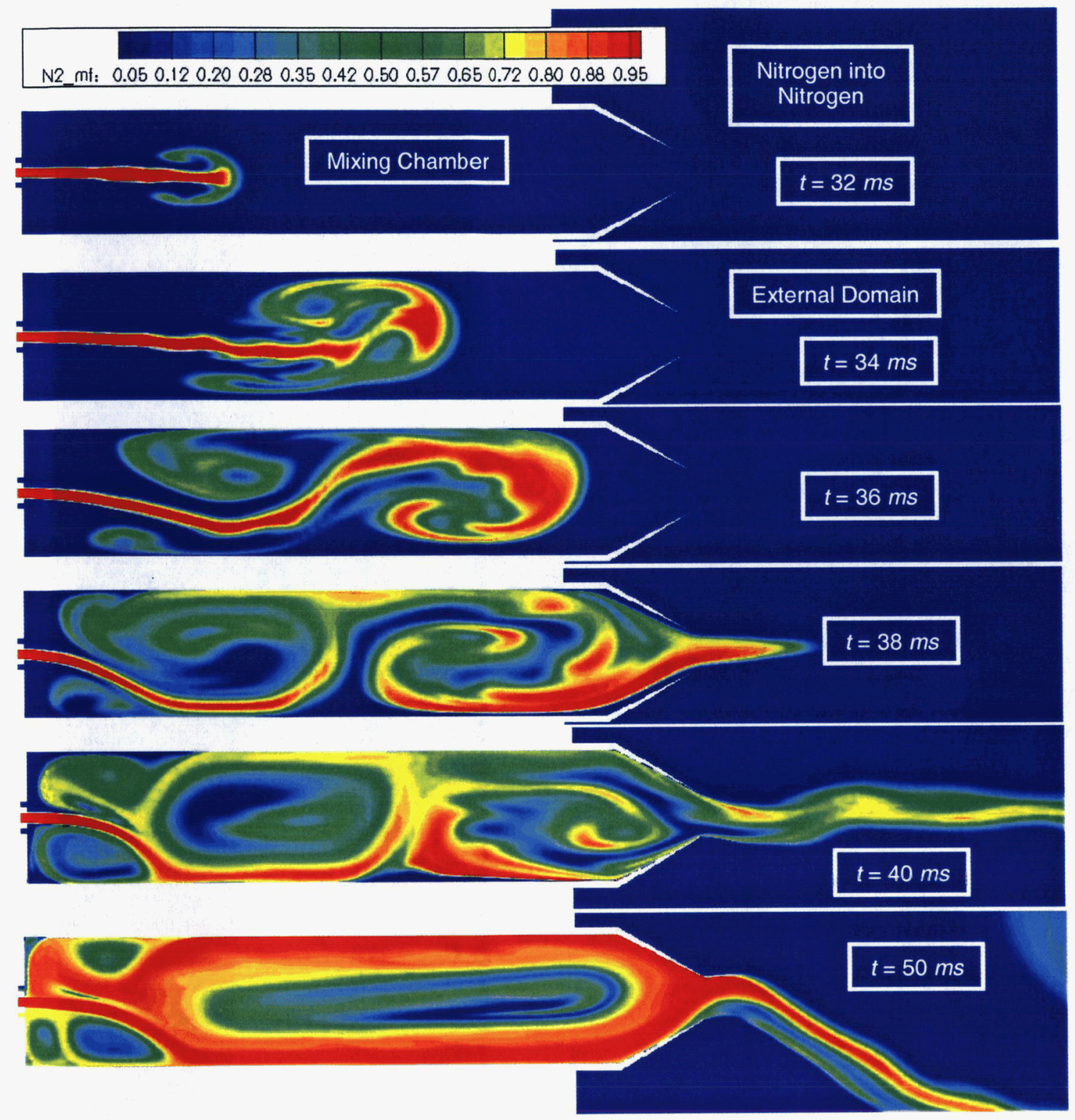

Figure 9. Various times of transient mixing of nitrogen injected into nitrogen.

\section{Comparison with Experiment}

Comparisons between the numerical simulations and quantitative planar laser-induced fluorescence (PLIF) images of the nitrogen concentration ${ }^{1}$ are shown in Figs. 10 to 13. The color scales for the concentration contours in the computations and experiments are given at the top of each figure. Note that sequences in the color scales between computation and experiment are different. The experimental results show the mass fraction of nitrogen, whereas the computational results show the mass fraction of helium. In the experiments, yellow indicates a maximum concentration of nitrogen, while black indicates a maximum concentration of helium. In the computations, blue indicated a maximum concentration of nitrogen, while red indicates a maximum concentration of helium. 


\section{Computation (Mass Fraction of $\mathrm{He}$ )}
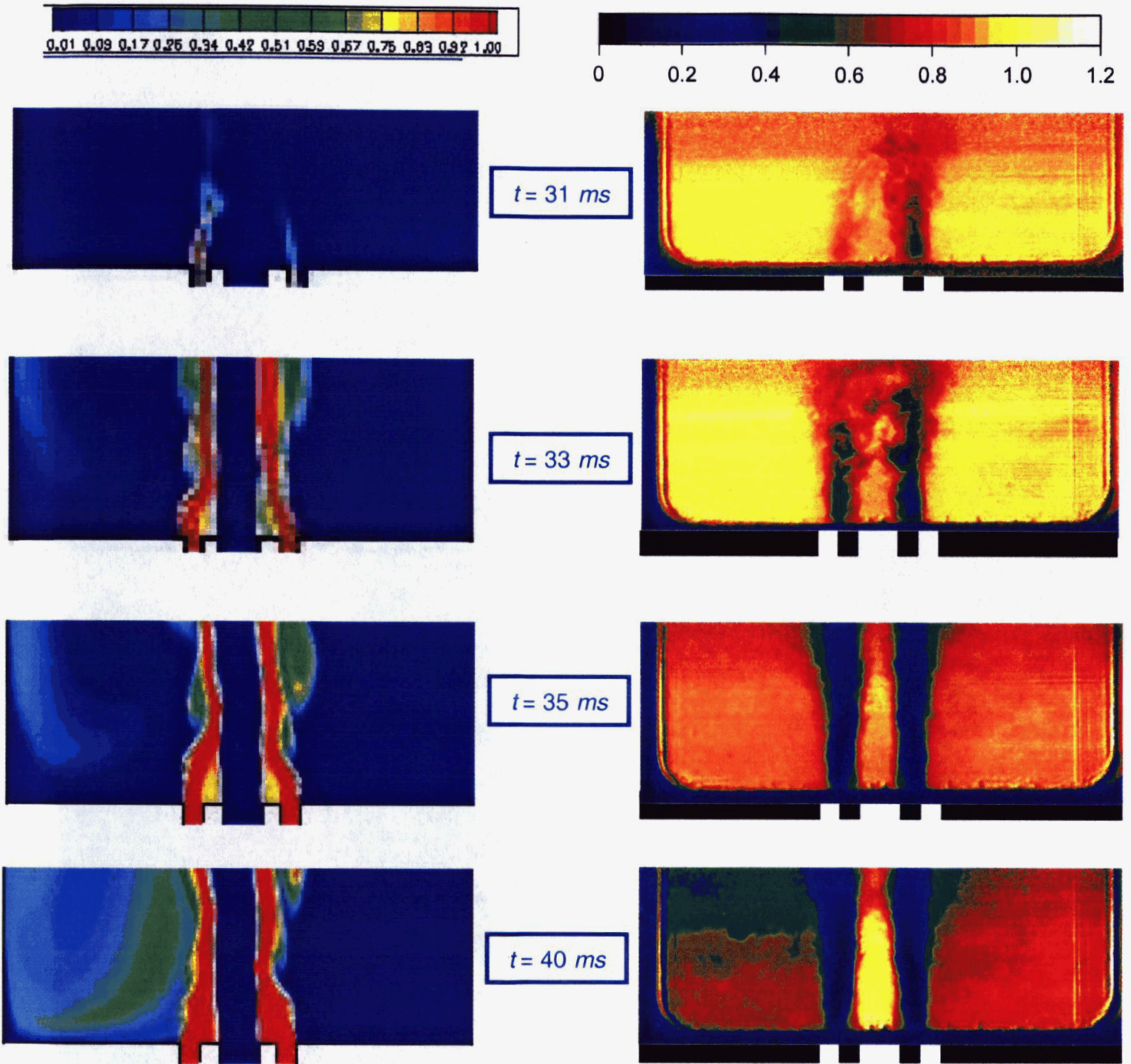

Figure 10. Comparison of $\mathrm{N}_{2}$ / He contours adjacent to injector face for various times. Left: Computation mass fractions; Right: Averaged Experimental PLIF Measurements. Flow is from bottom to top.

The results in Fig. 10 show a $10 \mathrm{~mm}$ region immediately adjacent to the injector face that spans the 38 $\mathrm{mm}$ width of the chamber including the two helium streams, the nitrogen stream and the adjoining base region extending to the chamber walls. Snapshots of the transient mixing process in this region are given at four specific times, $t=31,33,35$, and $40 \mathrm{~ms}$, after the helium valve has been opened. The column on the left shows the computational predictions, while that on the right shows the experimental images. A total of four separate instantaneous images has been averaged to generate the experimental results. At $t=31 \mathrm{~ms}$, the helium has just started to enter the chamber in both the experiments and the computations. In the experimental visualization, the helium jet on the right emerges first, while in the computations, the one on the left emerges first. The delay between the two is approximately $1 \mathrm{~ms}$ in both cases. As noted earlier, the delay in the computations occurs because of the modestly lower pressure in the small recirculation zone on the attachment side of the nitrogen jet. The reason for the similar delay in the experiment is uncertain, but may have occurred for the same reason. At succeeding times, the flow from both helium streams is clearly 
visible with qualitative agreement between computation and experiment as to their location and shape. Note that the curvature of
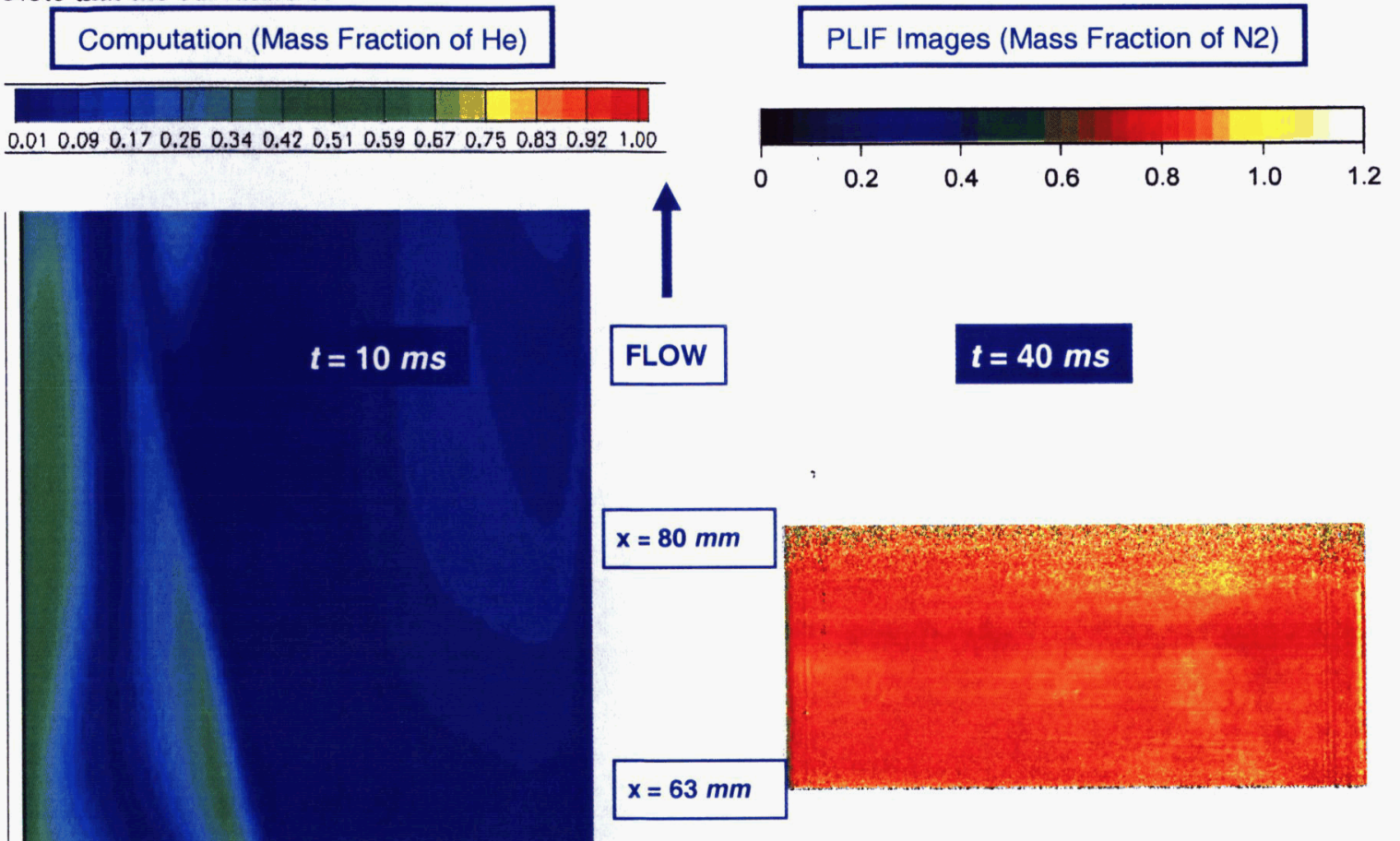

PLIF Images (Mass Fraction of N2)

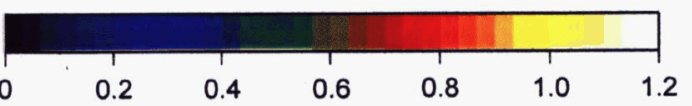

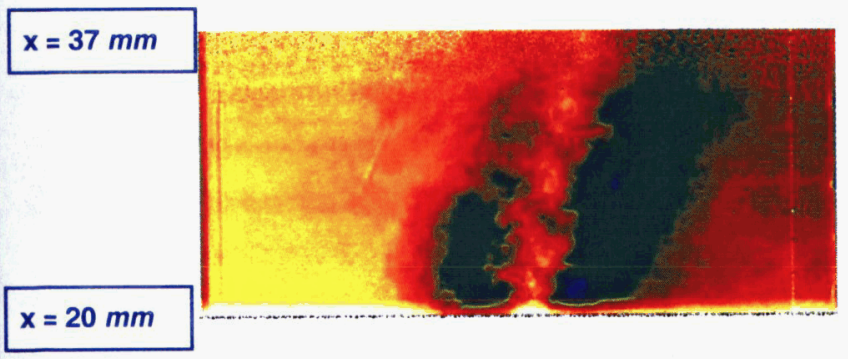

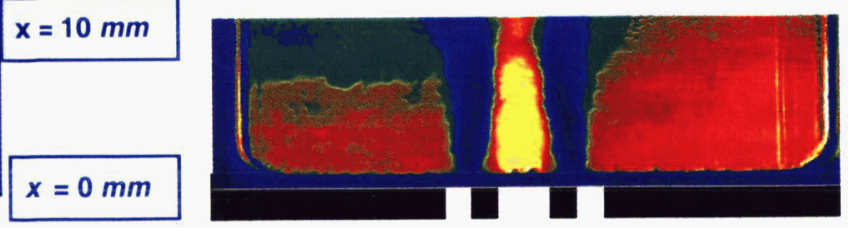

Figure 11. Comparison of computations and experimental flow visualization at $t=$ $10 \mathrm{~ms}$ after He begins to enter chamber. Left: Computational Mass Fractions; Right: Averaged Experimental PLIF Contours. 
the helium and nitrogen jets to attach to the wall as shown in Fig. 7 is not visible in the present-snapshots because of the relatively short stream wise extent. Both the experiment and the computational results indicate that the recirculation zone on one side of the incoming jets (in the computation, it is the side to which the nitrogen jet attaches as was noted earlier) begins to fill with helium at approximately the same time (between $t=35$ and $40 \mathrm{~ms}$ ).

Figs. 11 and 12 compare experimental images at three locations inside the chamber with computational predictions for the entire domain at times $t=40 \mathrm{~ms}$ and $t=50 \mathrm{~ms}$, respectively. The three computational locations include the same $10 \mathrm{~mm}$ section adjacent to the injector face as shown in Fig. 10, along with two additional sections, each $17 \mathrm{~mm}$ high, positioned between 20 and $37 \mathrm{~mm}$, and 63 and $80 \mathrm{~mm}$ above the face. The corresponding computational results cover the first $100 \mathrm{~mm}$ of the chamber. In general, there is again reasonable qualitative comparison between the computation and the experiment at both times.

At the earlier time $(40 \mathrm{~ms})$ in Fig. 11, the comparison at the lower position is reasonably quantitative as noted in the discussion of Fig. 10, while at the later time $(50 \mathrm{~ms})$ the experiment shows that the recirculation regions on both sides of the jet are filled with helium whereas the computations indicate that one side remains pure nitrogen. This difference is probably attributed to three-dimensional effects as noted in the next section. The comparison at the middle location indicates an asymmetric concentration of helium in both computation and experiment with a clearly discernible nitrogen stream between helium patches at $t$ $=40 \mathrm{~ms}$ (Fig. 11) suggesting that the experimental jet is adjacent to the wall as noted earlier in the computational discussion. The plots at $t=50 \mathrm{~ms}$ (Fig. 12) show substantial amounts of helium on both sides of the jet with the experiment perhaps indicating more rapid mixing, but both suggest an asymmetry remains.

The experimental images at the top-most location indicate that the flow is nearly pure nitrogen. While the computations show regions of helium are present, it is relatively dispersed. Overall, even at this location, the agreement is not unreasonable.

As a final two-dimensional comparison between the computations and the experiments, we show results at a sequence of four times, $t=35,40,50$ and $65 \mathrm{~ms}$, in Fig. 13. The field of view here starts from the injector face and extends a distance of approximately $100 \mathrm{~mm}$. The flow structure predicted by the computation is qualitatively similar to the experimental images. The experimental images clearly show that the jet is inclined from the center line and suggests that the helium concentration is larger on the side of the jet closest to the wall. In general, the experiment indicates that the nitrogen stream bends toward different sides in different runs. It also appears that the computation somewhat over-predicts the curvature in the jet. The computational jet is consistently closer to the wall than the experimental images indicate, and at later times, the experimental jet becomes nearly symmetric whereas the computational solution has moved farther from the wall, but still exhibits asymmetry. There are also differences between the simulation and experiment in the local details of the shear layer interface between nitrogen and helium jets. In the computations the nitrogen stream is not interrupted by the helium streams as is observed in the experiment. Nevertheless, the overall comparison with experiments is very encouraging in that the dominant features are predicted correctly in a qualitative manner. 

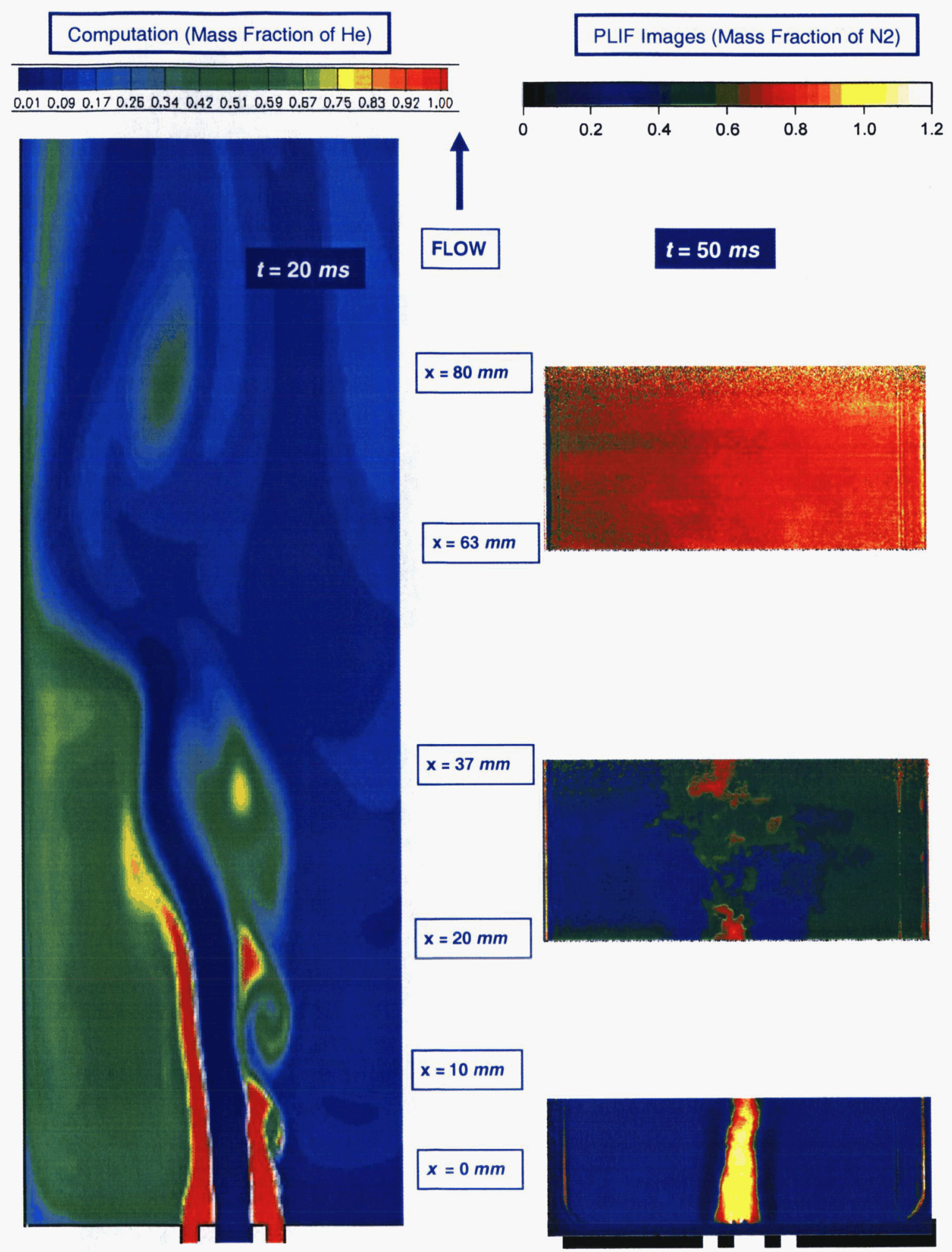

Figure 12. Comparison of computations and experimental flow visualization at $t=$ $10 \mathrm{~ms}$ after He begins to enter chamber. Left: Computational Mass Fractions; Right: Averaged Experimental PLIF Contours. 
Fig 13 missing

\section{Three-Dimensional Transient Helium/Nitrogen Mixing Simulations}

The two-dimensional solutions indicated that the flow in the chamber was often characterized by asymmetry. Because the chamber with height 0.75 " yielded both symmetric and unsymmetric solutions, this configuration was chosen for further computational consideration in three dimensions. A 3-D computational grid was generated by stacking the two-dimensional grid with stretching near the end-walls to accommodate the boundary layers. A total of 50 span-wise planes were used resulting in 3,900,000 nodes. Three-dimensional calculations were carried out for two inlet flow Mach numbers, 0.1 and 0.53 , corresponding to the reduced and base mass flow conditions from the 2-D solutions respectively.

A much stronger three-dimensional effect was observed when the flow rate was increased to the base flow rate. Fig. 14 shows a three dimensional view of the Mach number contours at six stream wise planes. As the jet moved downstream, it first attached to the lower wall in much the same manner as in the 2D calculations, but then shifted toward one corner, creating a large 3D circulation region. The corresponding contour plots at various span-wise locations are given in Fig. 15. The Mach number contours appear much like the 2-D results in the region near the injector face, but near the nozzle the 3-D asymmetries clearly appear. Again, these solutions suggest that three dimensional effects do not provide an overwhelming argument for symmetry, but rather present additional avenues through which asymmetry can enter.

\section{Conclusions}

In this paper, we presented the results of two-dimensional modeling of the unsteady cold flow mixing of gases discharged from injector slots into a common mixing chamber.

\section{Summary and Conclusions}

Numerical simulations of the unsteady mixing between two dissimilar gas streams emerging from separate, fully developed turbulent channel flows into a common mixing chamber were presented. The geometry and flow conditions of interest were chosen to match conditions in a companion experimental program. Nitrogen and helium, with a molecular weight ratio of seven, were used as the working fluids to simulate mixing in the pre-ignition environment in a liquid propellant rocket engine. The geometrical configuration was set up to provide two-dimensional mixing, although there are clearly three-dimensional effects present. As an initial step in understanding the physics of the unsteady mixing process, most of the computations have been two-dimensional, although some preliminary three-dimensional solutions have been obtained. The key area of interest was the transient mixing between an established, steady flow of nitrogen into which a helium stream is injected. The gases were injected into the chamber through three slots with the nitrogen in the middle and a helium slot on either side. Calculations were carried out for both the symmetric case and the unsymmetric case. For the symmetric case, the centerline of the nitrogen stream was treated as a symmetry plane and only one helium stream was modeled. For the unsymmetric case, the flow from all three slots was modeled and the nitrogen stream was allowed to move off the centerline.

The two-dimensional steady flow results for nitrogen injection in the absence of helium suggested that obtaining a symmetric flow in the chamber at the Reynolds numbers of interest (nominally 40,000) was unlikely. In efforts to find a symmetric steady flow solution, the chamber height was decreased by four, the mass flow was decreased by a factor of five and the external pressure was reduced by a factor of five. In most cases the flow still deviated from the centerline and attached to one side wall. Some isolated 
conditions were found for which the nitrogen jet remained on the centerline. Computations in the symmetric chamber attached randomly to either side, but having once attached were very stable. -

A three-dimensional computation for the configuration that produced the 2-D symmetric solution indicated that the same mass flow produced a nearly symmetrical solution that exhibited weak 3-D effects. Increasing the mass flow rate to the nominal value produced a jet that initially attached to one side wall and then shifted toward the end to produce a non-uniform flow in the span-wise as well as the cross-stream direction.

Unsteady flow calculations in which a helium stream was injected into the above-described, steady nitrogen flows were carried out for several configurations. For the nominal configuration the calculations suggested that the concentration of fuel increased much more rapidly in the closed recirculation region on the side where the jet was attached. Nevertheless, filling this 'dead water' region took nearly $100 \mathrm{~ms}$. The time required to fill the large recirculation region on the opposite side was much longer and was not simulated. The unsteady mixing process initially showed strong vortex rollup between the injected helium and the nearly quiescent adjacent nitrogen in the recirculating flows, but as the fluid in the recirculating region was replaced by helium, this vortex rollup decreased and a smooth shear layer was generated.

Comparisons between the computations and experimental flow visualizations indicated that the computations provided a fair prediction of the flow, but the experiment indicated mixing rates that were considerably faster than those predicted in the computations. In particular, the experiments showed that the nitrogen in the both corners of the chamber was replaced by helium in approximately the same time, while the computations indicated the nitrogen in only one corner was replaced as quickly. The entering flow near the injector face was, however, predicted quite well, but the nitrogen jet disintegrated more rapidly in the experimental flow visualizations than in the computations. Three-dimensional flow solutions are currently being obtained to assess the impact of three-dimensionality on the predictions. 

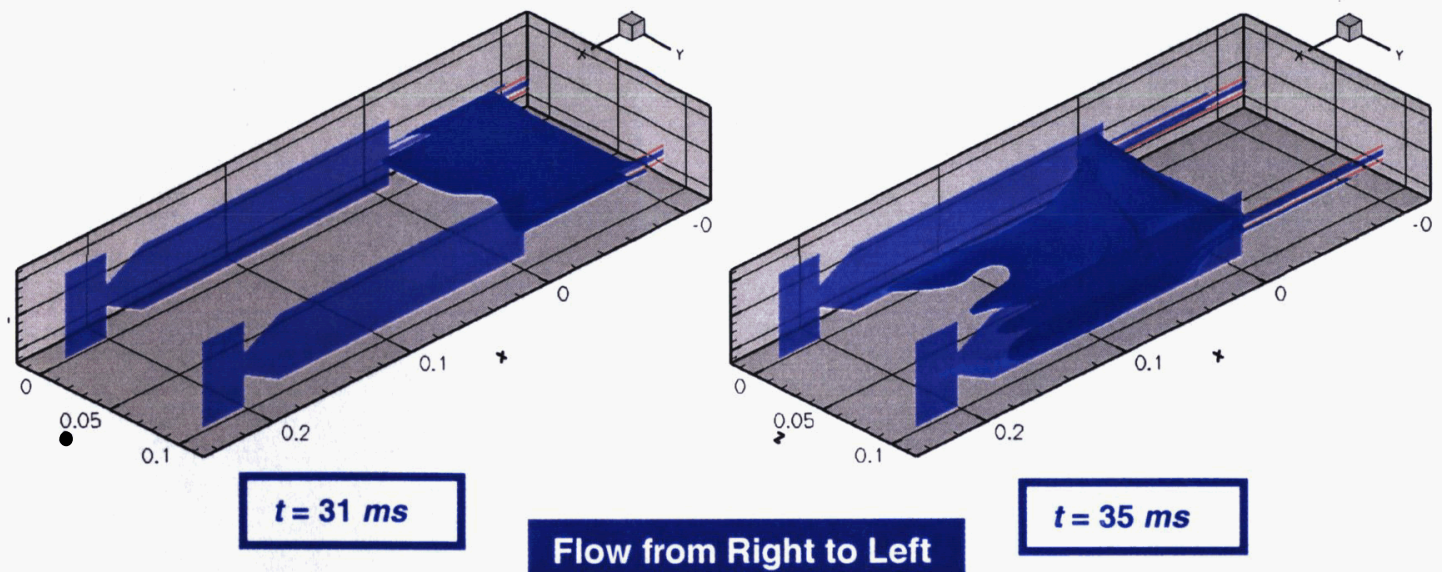

Flow from Right to Left
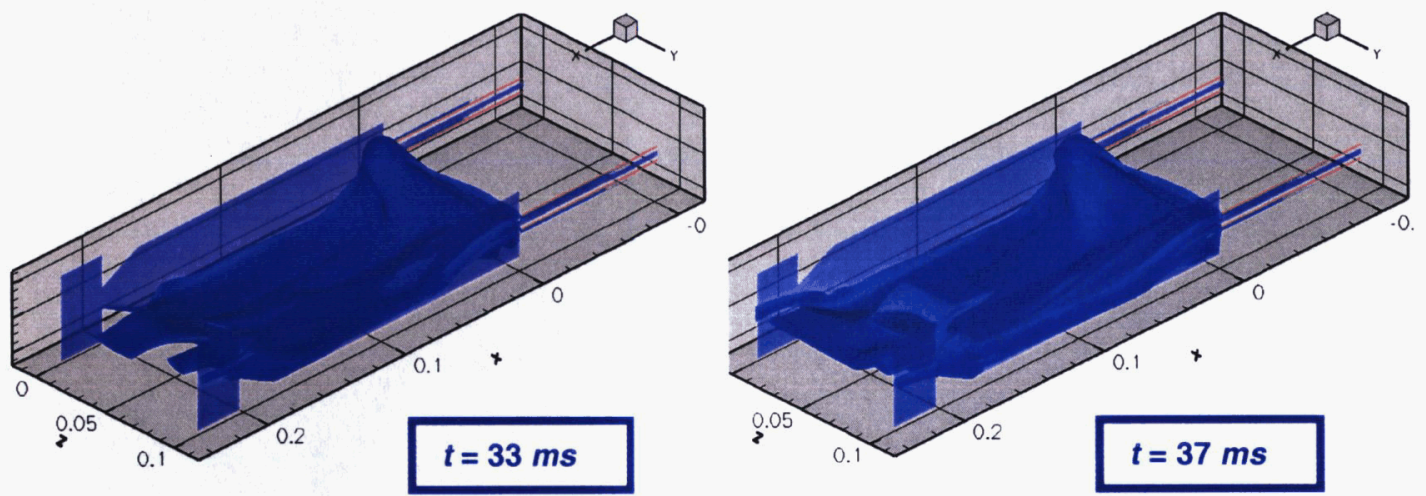

Figure 14. : Instantaneous concentration iso-surfaces of $\mathrm{He} \mathrm{c}=0.1$ at different times. Central channel Mach=0.63, Side channels 0.35. 


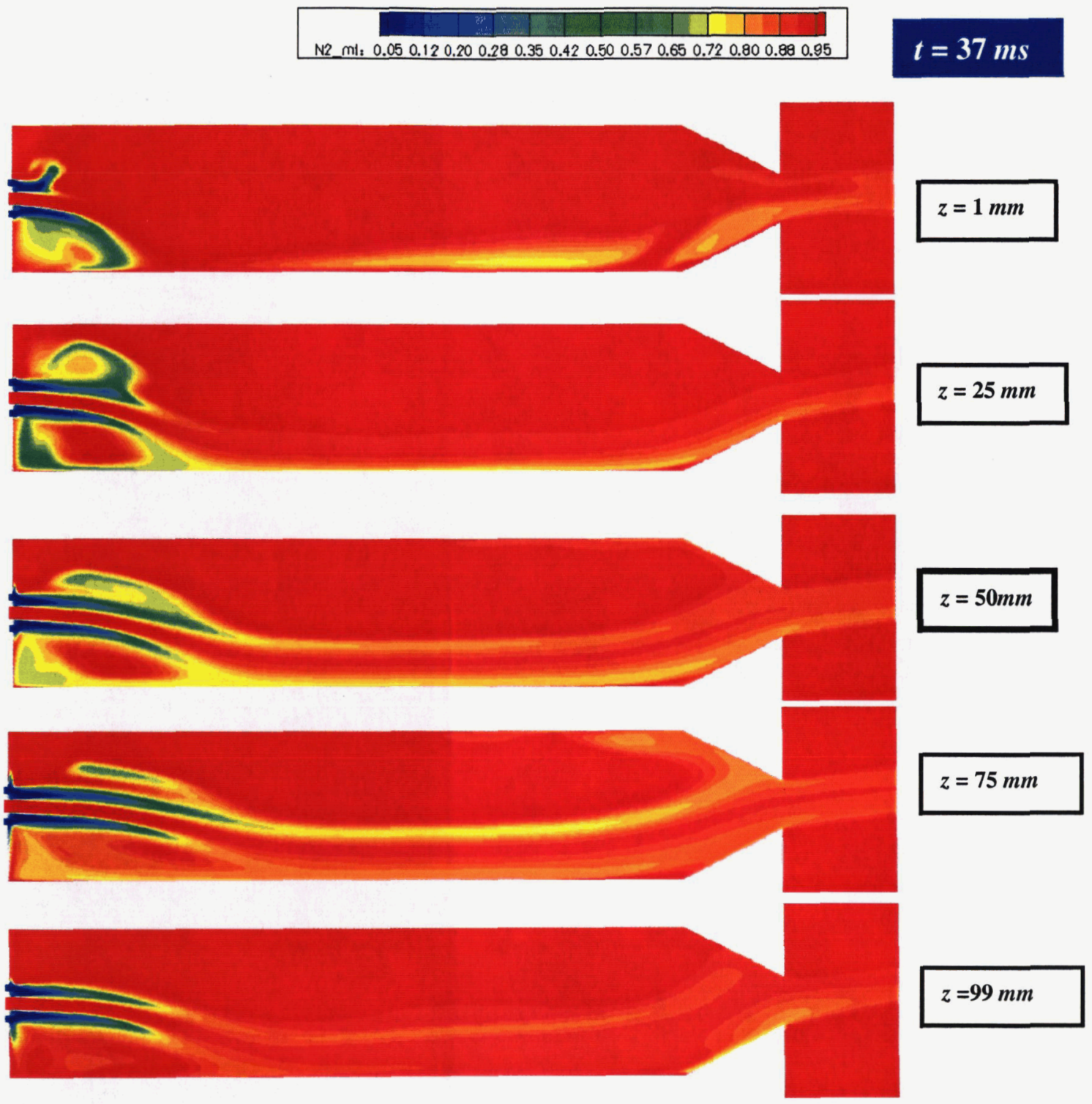

Figure 15: Instantaneous concentration contours at $\mathbf{t}=\mathbf{3 7} \mathrm{ms}$ at various span wise locations 


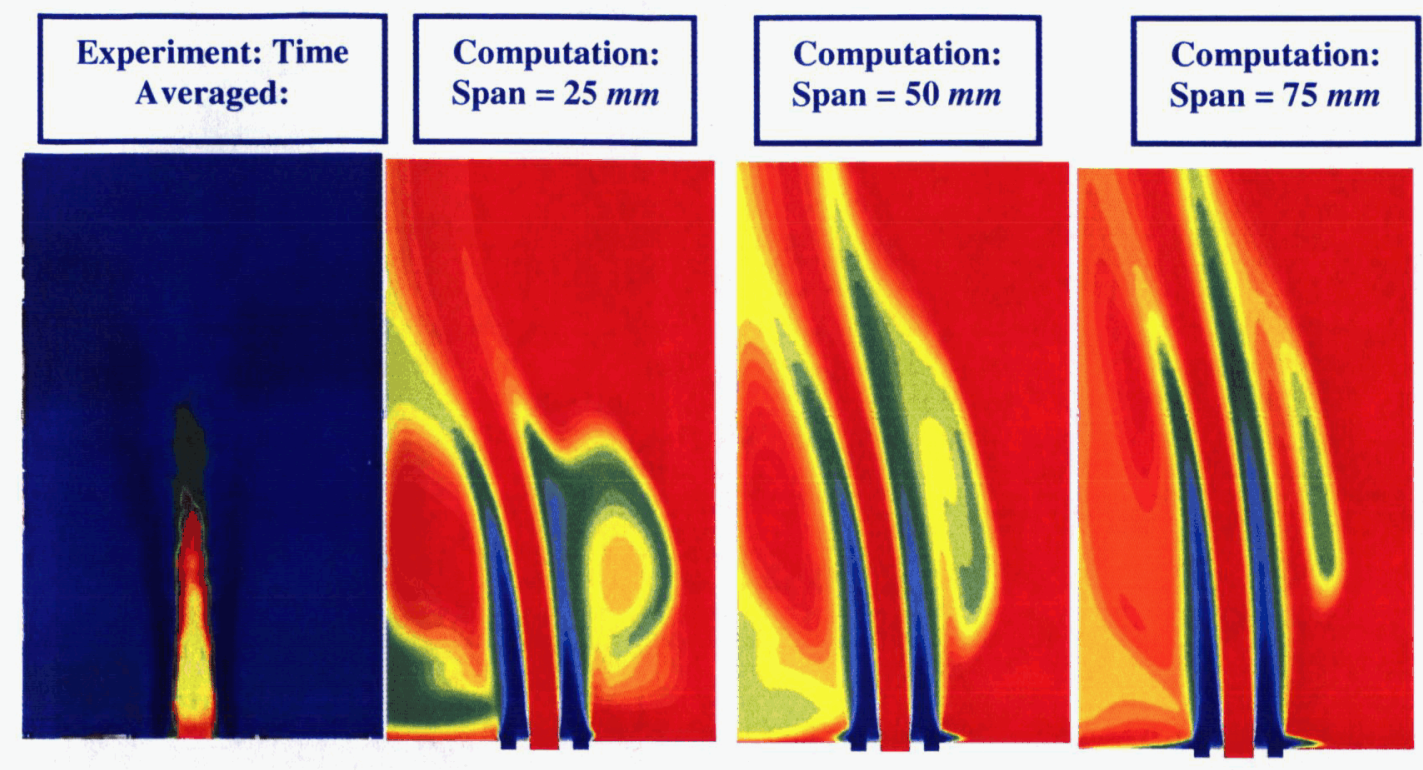

Figure 16. Span-wise cuts of helium concentration contours. Comparison with experiment.

\title{
VI. Summary and Conclusions
}

\section{Acknowledgments}

\begin{abstract}
References
${ }^{1}$ Lucht, R.P., Kulatilaka, W., Tseng, C., Merkle, C., Hulka, J.R., and Jones, G., 'Cold Flow Mixing Simulation Experiments for Liquid Rocket Chambers,' AIAA Paper No. 2006-4530, July 2006.

${ }^{1}$ Lucht, R.P., Sisco, J.C., Meyer, S.E., Anderson, W.E., Jones, G., and Hulka, J.R., 'Bench-Scale Cold Flow Simulant Mixing Experiments of Liquid Propellant Rocket Engine Ignition,' $52^{\text {nd }}$ JANNAF Propulsion Meeting, Monterrey CA, Dec. 5-8, 2005.

${ }^{2}$ Schmidt, V., Klimenko, D., Haidn, O., Oschwald, M., Nicole, A., Ordonneau, G., and Habiballah, M., 'Experimental Investigation and Modeling of the Ignition Transient of a Coaxial H2/O2-Injector; $5^{\text {th }}$ International Symposium on Space Propulsion, Oct. 27-30, 2003, Chattanooga, TN.

${ }^{3}$ C. Hensel, D. Wiemann, W. Oechslein, J, Görgen, 'Ignition Aspects for the Vinci Thrust Chamber,' AIAA 20024008.

${ }^{4}$ K. McManus, F. Aguerre, B. Yip, S. Candel, 'Analysis of the Ignition Sequence of a Multiple Injector Combustor Using PLIF Imaging, Non Intrusive Combustion Diagnostics,' K, Kuo, T. Parr (Eds.), Begell House, 1994.

6 Sarma A.S.R., Sundararajan T. and Ramjee V., "Numerical Simulation of Confined Laminar Flows," International Journal for Numerical Methods in Fluids, 2000, 33, 609-626.

${ }^{7}$ Back L.H., Roschke E.J., "Shear-Layer Flow Regimes and Wave Instabilities and Reattachment Lengths Downstream of an Abrupt Circular Channel Expansion," Transactions of the ASME, Journal of Applied Mechanics, Vol 39, Nov 3, 1972.

${ }^{8}$ Wilcox, D.C., Turbulence Modeling for CFD, $2^{\text {nd }}$ Ed., DCW Industries, La Canada, CA, 1998.

Gupta reno paper

${ }^{9} \mathrm{Li}$, D., Xia, G. and Merkle, C.L., "Analysis of Real Fluid Flows in Converging Diverging Nozzles," AIAA Paper 2003-4132, 2003.

${ }^{10}$ Sankaran, V. and Merkle, C.L., "Artificial Dissipation Control for Unsteady Computations," AIAA Paper 2003-3695, 2003.

${ }^{11}$ Experimental paper with asymmetric flow at low Reynolds number (Dima)
\end{abstract}


${ }^{12}$ West, J., NASA Marshall Space Flight Center, Personal Communication, Jan. 2006. 